\title{
Prevention of postoperative pulmonary complications and aggregation of marginal gains
}

\author{
Amedeo Anselmi, MD, PhD, and Jean-Philippe Verhoye, $\mathrm{MD}, \mathrm{PhD}$
}

\footnotetext{
From the Division of Thoracic and Cardiovascular Surgery, Pontchaillou University Hospital, Rennes, France. Disclosures: Authors have nothing to disclose with regard to commercial support.

Received for publication Oct 21, 2016; accepted for publication Oct 21, 2016; available ahead of print Nov 19 , 2016.

Address for reprints: Amedeo Anselmi, MD, PhD, Division of Cardiovascular and Thoracic Surgery, Pontchaillou University Hospital, 2 rue Henri le Guilloux, 35000 Rennes, France (E-mail: amedeo.anselmi@alice.it). J Thorac Cardiovasc Surg 2017;153:735-6

$0022-5223 / \$ 36.00$

Copyright (C) 2016 by The American Association for Thoracic Surgery

http://dx.doi.org/10.1016/j.jtcvs.2016.10.049
}

Systemic inflammatory reaction associated with cardiopulmonary bypass (CPB), along with its manifestations on various systems and end-organ function, are among the most studied topics in cardiac surgery. ${ }^{1}$ The postoperative recovery of lung function strongly determines the clinical success of cardiac operations; yet the lung tissue is a primary target for CPB-induced injury, as the result of complex interactions among immunologic, mechanical, and hemodynamic contributors. The clinical implications of such injury are mostly evident in patients with underlying pulmonary comorbidities. Postoperative mortality can be tenfold higher in patients with preoperative moderate or severe airway obstruction or with diffusing capacity for carbon monoxide $<50 \%$ than predicted. ${ }^{2}$ Thus, preoperative lung disease represents a relatively frequent reason for surgical denial.

In this issue of the Journal, Taki and coworkers ${ }^{3}$ report that administration of allogenic fetal membrane mesenchymal stem cells (FM-MSCs) may reduce cytokine (eg, TNF- $\alpha$, IL-6, IL-1 $\beta$ ) expression both in serum and lung tissue, and significantly decrease lung tissue injury through preservation of alveolar integrity and minimization of interstitial neutrophil infiltration. Some aspects of their rat CPB model can be ameliorated and the experiments repeated under conditions more similar to the clinical setting (eg, full-flow and longer CPB, mild or moderate hypothermia) with a more complete dataset (eg, multiple serum cytokine measurements) before planning a human trial. Other elements to be addressed are the immunogenicity of FM-MSCs and the possibility obtaining them from placentas after delivery without harming human embryos or fetuses. Nonetheless, the authors' results are of major interest in the multidisciplinary efforts toward improved organ protection in cardiac surgery. Such efforts are ultimately aimed allowing patients with more severe disease to enjoy the reproducible and durable benefits of valvular/coronary/ aortic operations.

Can we imagine that in the future, transplantation of allogeneic FM-MSCs will be part of a multimodal

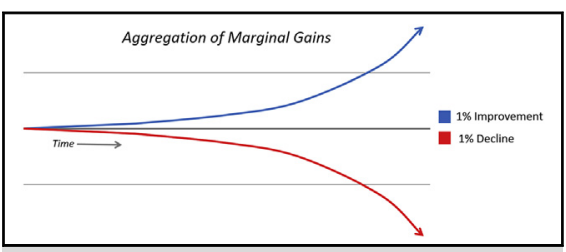

The concept of aggregation of marginal gains.

\section{Central Message}

Allogeneic fetal membrane mesenchymal stem cell transplantation can be further evaluated as an additional measure to minimize cardiopulmonary bypass-induced lung injury, and potentially included into multimodal protocols for clinically overt gains.

See Article page 726.

approach to minimize lung tissue injury and optimize postoperative pulmonary function, together with other strategies such as continued ventilation during CPB, preoperative pulmonary conditioning, and minimally invasive surgical access (and others)? Although none of these strategies taken individually is likely to confer a major advantage in terms of operative risk, some combination is likely needed to provide a significant impact that translates into clinically measurable outcomes (eg, minimal rates of prolonged ventilation and postoperative tracheostomy, possibility of achieving fast-track recovery). In this respect, one might evoke the concept of the aggregation of marginal gains (Figure 1).

Initially described in the context of competition cycling, this concept is indeed applicable to many complex social sciences, including management of an institutional cardiothoracic surgery program. Essentially, the progressive accumulation of many minor changes in the correct direction will ultimately lead to a major gain, even though the

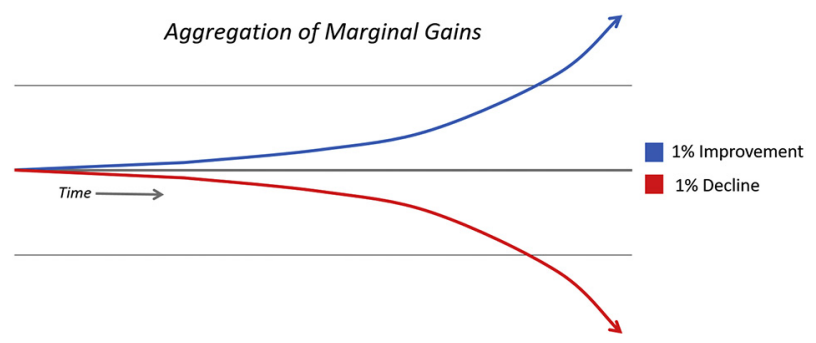

FIGURE 1. The concept of aggregation of marginal gains. 
immediate single benefit is small. In the current era, characterized by continuing worsening of the baseline profile of surgical candidates as well as by the sometimes difficult choices among surgical and alternative transcatheter techniques, there is an ongoing need to further decrease the invasiveness of surgery while maintaining its reproducibility. Regardless of the approach chosen, preparation needs to be done with the same meticulous approach as for Olympic cycling.

\section{References}

1. Clive Landis R, Murkin JM, Stump DA, Baker RA, Arrowsmith JE, De Somer F, et al. Consensus statement: minimal criteria for reporting the systemic inflammatory response to cardiopulmonary bypass. Heart Surg Forum. 2010;13: E116-23.

2. Adabag AS, Wassif HS, Rice K, Mithani S, Johnson D, Bonawitz-Conlin J, et al. Preoperative pulmonary function and mortality after cardiac surgery. Am Heart J. 2010;159:691-7.

3. Taki T, Masumoto H, Funamoto M, Minakata K, Yamazaki K, Ikeda T, et al. Fetal mesenchymal stem cells ameliorate acute lung injury in a rat cardiopulmonary bypass model. J Thorac Cardiovasc Surg. 2017;153:726-34. 